\title{
Research on the Application of Concept Map in Professional Knowledge Structure of Post-oriented Students
}

\author{
Jianfeng Chen \\ Graduate Students Brigade, Logistics University of PAP \\ Tianjin, 300309 China
}

\author{
Yu Zhang* \\ Logistics support Department, Logistics University of PAP \\ Tianjin, 300309 China
}

\begin{abstract}
Starts with the importance of concept map in evaluating professional knowledge structure of PAP postoriented students and combines with the advantages of concept map evaluation, this paper describes aspects of concept map, such as knowledge structure evaluation method, questionnaire design, and testing and evaluation process, then analyzes the test result, and finally puts forward the feasibility and validity of concept map in evaluating professional knowledge structure in order to provide reference and analysis tool for further optimization of teaching resources and models of post-oriented education.
\end{abstract}

Keywords-concept map; post-oriented students; professional knowledge structure; evaluation

\section{INTRODUCTION}

On the basis of educational background and adapting to the specific post requirements of the Armed Police Force, the post education of the Armed Police Force is a practical activity of carrying out professional knowledge, professional ability and professional accomplishment education for the active serving Armed Police officers with certain positions ${ }^{[1]}$. It is a practical educational activity with the demand of post and post as its traction and ability training as its basis. Compared with academic education, it pays more attention to the cultivation of professional quality. And one of the core indicators of police officers' professional quality is professional knowledge structure ${ }^{[2]}$, which is the basis of professional quality and plays a vital role in the formation of police officers' quality. Under the guidance of headquarters, colleges and universities carry out on-the-job training, pre-job training, professional training and other on-the-job education for serving students, in order to enable them to have the corresponding professional knowledge structure, with a view to forming the basis of post professional competence. Then, how to judge what kind of professional knowledge structure the trainees have formed after training. Accordingly, adjusting and optimizing teaching resources and modes has become a key issue affecting the quality of Vocational education.

At present, after training, rotating training or intensive training, the trainees usually use the form of examinations to examine their knowledge structure. The main types of examination questions are objective questions and subjective questions, which have inherent drawbacks. First of all, objective questions are in the form of blank-filling questions and multiple-choice questions. When students are ambiguous about a certain knowledge point, they may unconsciously choose the correct answer, which makes it difficult to accurately distinguish the students' mastery of knowledge points. Concurrently, the knowledge points of objective questions are often scattered, and it is difficult to know the overall situation of students' knowledge construction. Secondly, subjective questions appear in the form of questions and answers or thesis writing. Although subjective questions can more or less understand the students' knowledge structure, they are not comprehensive enough. Moreover, it is more difficult for students to measure their knowledge structure when they rush to memorize or find someone to write essays in the past. Generally speaking, it is difficult to effectively adjust teaching resources and modes to meet the development and needs of vocational education by examining students' knowledge points and knowledge structure in the traditional examination form without quantitative analysis and visual representation.

Compared with the drawbacks of traditional examinations, the assessment model based on concept map has the advantages of quantitative analysis and visual evaluation. Firstly, the professional knowledge structure established consciously or unconsciously by the students through vocational education is a relatively static knowledge node network, which can be represented by the form of concept map (proposition and schema). Proposition is the smallest unit of meaning, which consists of a set of conceptual nodes and connections. At the same time, if there is a common component (concept node) among different propositions, then we can rely on this common component to connect several different propositions through "branch, level, horizontal connection", thus forming a propositional network, which can be effectively displayed in the form of schema. On this basis, combined with the evaluation index system, we can quantitatively evaluate the degree of knowledge understanding of the respondents. In addition, the concept map is a visual form of graphics, in which the skyline between concepts can be clearly drawn, and the concept can also be continuously 
expanded, which can directly reflect the cognitive level and knowledge construction status of the subjects. Therefore, concept maps can be an effective tool for assessing the professional knowledge structure of serving trainees.

\section{THE THEORETICAL STUDY ON THE STRUCTURE OF PROFESSIONAL KNOWLEDGE OF SERVING TRAINEES BASED ON CONCEPT MAPS}

To evaluate the professional knowledge structure of the trainees based on concept maps, theoretical research is first involved, which mainly includes the following three aspects:

\section{A. Resource Selection of Standard Concept Map}

In terms of resource selection, first of all, we should embody the universality of resources, ensure that resources have sufficient breadth, and avoid excessive expansion. For example, the selection of resources for the standard concept map of combat cadets mainly focuses on four aspects of literature: combat readiness, training, management and political work. Secondly, in order to adapt to the specialty and post characteristics of the respondents, experts should choose the specialty literature suitable for their specialty and post when choosing resources, and understand the commonly used professional terms. Thirdly, explanatory text is the main resource choice, which facilitates the extraction of concept nodes. The selection of resources can be divided into four steps: (1) To determine the subject of resources and the participants, first of all, the subject content of the selected resources should be clearly defined, and four experts in professional fields and several evaluation designers should be identified. (2) To determine the criteria for resource selection, experts and designers are required to independently select and arrange 10 optimal resources. (3) Through paired combination, experts and designers can choose 20-40 most suitable funds for thematic content cooperation Source. (4) Four experts cooperated to review the resources selected in the third step and form the final 20 resources.

\section{B. The Generation of Standard Concept Map}

In order to ensure the standardization of the standard concept map generation, it is necessary to hold expert training meetings before the generation process to ensure the consistency of each expert's work in the production process. The specific generation process is divided into three steps:

The first step is to establish the corresponding concept map for 20 selected resources separately. Experts are required to capture and present 20 resources using node concept maps. That is to extract conceptual nodes from resources, analyze and label the relationship between conceptual nodes, construct different propositions, divide conceptual map levels, and form a conceptual map corresponding to each resource.
The second step is to integrate 20 concept maps constructed by each expert into a single individual standard concept map. In the process of integration, three principles should be followed: firstly, the personal standard concept map should reflect 20 selected resources; secondly, the personal standard concept map should contain the concepts and relationships of key areas and explain their sources; thirdly, if the selected resources contain mutually contradictory concepts or propositions, it needs to be accurately calibrated. Inconsistence and root causes.

The third step is to form a standard conceptual map of the final related fields. In the final stage of completing the standard concept map, the individual standard concept map obtained by each expert in the second step is exchanged and discussed to determine the types and granularity of the concept nodes, the relationship between nodes, the correctness of propositions, record the differences of understanding in the individual concept map, and discuss the supplement or modification of the standard concept map by topic. In order to eventually form a standard concept map in related fields.

\section{Evaluation method of knowledge structure}

Knowledge structure evaluation is based on the standard concept map, using the corresponding evaluation methods, comparing and analyzing the personal drawings of the trainees, in order to judge whether the knowledge system of the trainees is comprehensive, whether the understanding of the concept nodes is correct, and whether the understanding of the relationship between the nodes (propositions) is correct. The main evaluation methods are: $\mathrm{C}$ technology $\mathrm{S}$ technology, F technology. C technology (construct-a-map with created concepts or linking phrases) ${ }^{[3]}$ is a creative concept map evaluation method, which extracts core concepts or corresponding concept series from the standard concept map and provides them to the trainees. The trainees determine the connection relationship, draw the concept map independently, and the judges compare it with the relevant parts of the standard concept map. Method of judgment. S technology (construct-a-map with selecledconcepts or linking phrases) ${ }^{[3]}$ is an evaluation method of selective concept maps, which extracts the whole or part of the conceptual map framework from the standard concept maps, in which several conceptual nodes or connection relationships are vacant, and provides the concept nodes or connection relationships that can be filled in. The trainees select the fillers and the judges. Comparing and evaluating the relevant parts of its standard concept map. F technology (fill-in-the-map) ${ }^{[3]}$ is an evaluation method for filling-in concept maps. Similar to S technology, the judges extract the whole or part of the concept map framework from their concept maps, in which there are several conceptual nodes or connections, but no options are provided. The participants fill in the relevant content and finally compare it with the relevant parts of the standard concept map. 


\section{Questionnaire Design, Testing AND Result ANALYSIS BASED ON CONCEPT MAP}

According to the results of theoretical research, the questionnaire based on concept map is usually designed as a composition questionnaire and a mapping questionnaire, which combines the structural scoring method and the relationship scoring method to test and evaluate the mastery of the professional knowledge structure of the incumbents.

\section{A. Questionnaire design}

\section{1) Composition of Questionnaire}

(1) Composition (Restricted) of Questionnaire

Teachers extract concepts and connective words from standard concept maps and ask students to create a concept map. For example, the concept nodes are given: logistics training, characteristics of logistics training, elements of logistics training, logistics training system, content of logistics training, organizational form of logistics training, etc. The connection relationship includes, has, divides and is determined by.. Students are required to make a concept map with these concept nodes and connections. This kind of questionnaire is difficult to some extent. It requires students to have a solid foundation and a comprehensive grasp of knowledge and its practical application. It is easier to detect the students' overall grasp of knowledge and inductive ability than the traditional test paper.

\section{(2)Composition (Unconfined)of Questionnaire}

Teachers extract children's main concepts from standard concept maps and ask students to construct a concept map. For example, conceptual nodes are given: logistical command, armed police logistical command, elements of armed police logistical command, stage division of emergency logistical command, logistical support preparation, etc. Students are required to use these conceptual nodes as the basis, and then select if from the designated data. To create a concept map by connecting concepts and concepts. This kind of questionnaire is innovative. Students can connect different concepts according to their work experience. It can detect the independent thinking and imagination of students better than the traditional test paper, and can stimulate their creativity.

\section{2) Map-filling Questionnaire}

(1) Mapping (S-type) Questionnaire

Teachers extract some conceptual maps from standard conceptual maps, leave out the blanks of conceptual nodes and connection relations, give options, and ask students to choose appropriate conceptual nodes and connection relations to fill in the blanks in the maps, which can be used to test the students' familiarity with the generation, connotation and extension of conceptual nodes. Such questionnaires are visually expressed. To reflect a large amount of knowledge information with fewer elements condensed in concept maps, and to use text language to the minimum, students can easily understand the relationship between concept nodes and strengthen the detection depth of knowledge structure.

\section{(2) Mapping (F-type) Questionnaire}

Teachers extract part of concept maps from standard concept maps and leave out the blanks of concept nodes and connection relationships. Unlike the S-type questionnaire, the questionnaire has no options, requiring students to create concept nodes and connection relationships independently. Filling in the blanks in the map with appropriate answers is basically the same as the S-type questionnaire, but relatively speaking, learning. Members have more freedom in answering questions. Because there is no guidance to choose the answer, students need to have a deeper understanding of the knowledge in the relevant fields, so it can reflect the students' understanding of the knowledge in the field more truly.

\section{B. Test and evaluation process}

\section{1) Training of trainees}

The vast majority of students have not been exposed to concept maps, so in order to ensure the validity of testing and evaluation, we first give them basic training. Using concrete and vivid examples to introduce the composition of concept maps to the trainees, enumerate the hierarchical and non-hierarchical examples of concept maps to analyze the types of concept maps, and let the trainees practice the construction of concept maps by themselves, master the basic rules and skills of drawing, understand the learning advantages of concept maps, such as visualization of knowledge structure, memory relevance, and stimulate their learning. Interest in learning.

\section{2) Organizing questionnaires}

Firstly, we selected 10 people from the two professional training courses, divided into group A and group B, 8 male students, 2 female students, 6 professional background, 4 other classics, the average age difference is less than 2 years old, the overall difference is no significant peer group, the questionnaire survey set up two raters. Secondly, group A students were given the composition (limited) questionnaire and the mapping (S) questionnaire in turn, and group B students were given the composition (nonlimited) questionnaire and the mapping $(\mathrm{F})$ questionnaire in turn. Closed-ended answers (40 minutes for the composition questionnaire, 20 minutes for the mapping questionnaire, 10 minutes for the interval) were adopted. During the period, students were not allowed to consult the designated data and exchange and test. Questions related to the trial task. Finally, the valid number of papers $(>90 \%)$ was collected for analysis.

\section{3) Organizing Questionnaire Evaluation}

The structure and relationship scoring method was used to compare with the standard concept map, and combined with the rater's evaluation, the questionnaire was evaluated. Composition questionnaires are scored by structure scoring 
method: every correct proposition (concept node and connection relationship are correct) is set as 1 point, every effective branch (proposition series) is set as 2 points, every effective level (same level branch) is set as 5 points, and every correct horizontal connection is set as 10 points, but for the correct but not clear concept relationship between the horizontal points. Directional connection is set at 2 points. The questionnaire was scored by relational scoring method: 0,1,2 points were used. The students did not answer or filled in blank items with complete errors as 0 points, and the answer was 0 points. Incomplete or partial errors are 1 point, and complete correctness is 2 points.

\section{CONCLUSION}

Through the analysis of questionnaires of two groups of students, it is found that: first, the performance of group A is slightly worse than that of group B, which is different from the anticipation. Group A questionnaires are less difficult than group B questionnaires, and they should be better. Probably the reason is that prompt questionnaires limit the independent thinking ability of students. The students focus on how to connect the given conceptual nodes into a whole while neglecting the divergent thinking developed by prompt nodes. Therefore, it is not mentioned in the future design of questionnaires. The proportion of demonstrative questionnaires was increased appropriately. Secondly, the total number of three components of concept maps drawn by high scorers in group A and B (mostly from their own professional background) is high, that is, the total number of concept nodes, connection relationships and effective connections is high. This shows that the concept maps drawn by students of different professional levels have significant differences, which is highlighted by the fact that high scorers have more professional knowledge reserves and can perform well. More correct conceptual nodes and links can be formed, and their links are more accurate. Thirdly, only $5 \%$ of the students in the two groups got high scores, and only $5 \%$ of the excellent ones (above 90 points) got high scores. Because the students seldom used "horizontal connection" correctly when drawing concept maps, resulting in a large number of scores loss, which indicates that the main line of knowledge structure of the textbook needs to be clear and perfect so that the students can grasp the relationship between the various knowledge points. Fourthly, most of the trainees constructed hierarchical concept maps. Only a few trainees constructed tree-shaped concept maps, and $75 \%$ of them were qualified for the evaluation (more than 60 points). This indicates that the trainees' knowledge of the structure of professional knowledge is at an elementary level and lack of innovative ability. It is necessary to further improve the teaching methods and improve the students' knowledge system. The ability to summarize.

\section{REFERENCES}

[1] Wang wei. Discussing the law of logistical education of armed police. Tianjin: Journal of Armed Police Logistics College (Comprehensive Edition), 2015, 3 (1): 48-50.

[2] Li Hao, Wu Shuai and Wang Gongbao. Comprehensive Quality Evaluation of Growing Cadre Students in Armed Police AcademyPrice Research [J]. Changsha: Literature and History Expo (Theory), 2012, (2) 47-49.

[3] Yue Yin, Jim Vanides, Maria Araceli Ruiz-Mapping Techniques: Implications for Scoring, of Research in science Teaching.2005, VOL.42 Primo. Comparison of Two Concepts Interpretation, and Use. Journal NO.2.166-184. 